\title{
Article \\ Evaluation of the Effect of Food Products Containing Prebiotics and Bacillus subtilis HU58 on the Gut Microbial Community Activity and Community Composition Using an In Vitro M-SHIME ${ }^{\circledR}$ Model
}

\author{
Massimo Marzorati ${ }^{1,2}$, Sarah Bubeck ${ }^{3, * \mathbb{D}}$, Thomas Bayne ${ }^{4}$, Kiran Krishnan ${ }^{4}$ and Aicacia Young ${ }^{4}$ \\ 1 Center for Microbial Ecology and Technology (CMET), Faculty of Bioscience Engineering, Ghent University, \\ Coupure Links 653, 9000 Ghent, Belgium; Massimo.Marzorati@prodigest.eu \\ 2 ProDigest, Technologiepark 82, 9052 Zwijnaarde, Belgium \\ 3 Bubeck Scientific Communications, 194 Rainbow Drive \#9418, Livingston, TX 77399, USA \\ 4 Microbiome Labs, 101 E Town Pl, Saint Augustine, FL 92092, USA; tom@microbiomelabs.com (T.B.); \\ kiran@microbiomelabs.com (K.K.); ayoung@microbiomelabs.com (A.Y.) \\ * Correspondence: bubeckscientific@gmail.com
}

\section{check for} updates

Citation: Marzorati, M.; Bubeck, S.; Bayne, T.; Krishnan, K.; Young, A. Evaluation of the Effect of Food Products Containing Prebiotics and Bacillus subtilis HU58 on the Gut Microbial Community Activity and Community Composition Using an In Vitro M-SHIME ${ }^{\circledR}$ Model. Appl. Sci. 2021, 11, 11963. https://doi.org/ 10.3390/app112411963

Academic Editor: Wojciech Kolanowski

Received: 10 November 2021

Accepted: 8 December 2021

Published: 15 December 2021

Publisher's Note: MDPI stays neutral with regard to jurisdictional claims in published maps and institutional affiliations.

Copyright: (c) 2021 by the authors. Licensee MDPI, Basel, Switzerland. This article is an open access article distributed under the terms and conditions of the Creative Commons Attribution (CC BY) license (https:/ / creativecommons.org/licenses/by/ $4.0 /)$.

\begin{abstract}
GoodBiome ${ }^{\mathrm{TM}}$ Foods is a collection of foods infused with prebiotics, including inulin and xylooligosaccharides, and the probiotic Bacillus subtilis HU58. The effects of repeated intake of three predigested GoodBiome ${ }^{\mathrm{TM}}$ Foods products and one comparator product on microbial community activity and composition were assessed using the mucosal simulator of the human intestinal microbial system (M-SHIME ${ }^{\circledR}$ ) platform with proximal colon (PC) and distal colon (DC) compartments and conducted under healthy gut conditions. Treatment with all test products increased short-chain fatty acid (SCFA) production (acetate, propionate, and butyrate) versus the control period in both the PC and DC. The highest increases were seen with the GoodBiome ${ }^{\mathrm{TM}}$ Foods products. Ammonium and branched SCFA levels were also increased (versus the control period) in both compartments. Treatment with all test products enhanced the Simpson diversity index (versus the control period), reaching significance for all test products in the PC $(p<0.05)$. Treatment with all test products resulted in changes in the microbial community composition. The relative abundance increased for Proteobacteria and decreased for Actinobacteria in the PC and DC. Repeated intake of GoodBiome ${ }^{\mathrm{TM}}$ Food products increased SCFA production and microbial diversity in an M-SHIME ${ }^{\circledR}$ model of the human intestinal microbiome.
\end{abstract}

Keywords: Bacillus subtilis; microbial community; prebiotic; probiotic; synbiotic

\section{Introduction}

Prebiotics have been defined by the International Scientific Association for Probiotics and Prebiotics as "a substrate that is selectively utilized by host microorganisms conferring a health benefit" [1]. These non-digestible oligosaccharides resist digestion and absorption in the human small intestine [2]. When they reach the large intestine, they are fermented by members of the gut microbiome and undergo complete or partial digestion; shortchain fatty acids (SCFAs) are a major product of their digestion [2,3]. Fermentation of prebiotics in the colon is usually quite rapid, particularly for short-chain prebiotics, and occurs mainly in the proximal colon (PC) [4-6]. This results in a higher concentration of butyrate in the PC than in the distal colon (DC) and may explain why there is a higher risk of adenocarcinoma in the DC versus the PC and that ulcerative colitis is localized to the DC [4]. The reported health benefits of prebiotics include increased SCFAs, modulation of the composition and function of the gut microbiome, improvement in immune function, and protective effects against the risk of colorectal cancer and its progression (reviewed in Davani-Davari et al. [3]). 
Probiotics, defined by The Food Agricultural Organization/World Health Organization as "live microorganisms which when administered in adequate amounts confer a health benefit to the host", are often consumed as functional foods or supplements. The benefits of probiotics may include manipulation of intestinal microbial communities, suppression of pathogens, immunomodulation, stimulation of epithelial proliferation and differentiation, and improvement of gut barrier function $[7,8]$.

Synbiotics are a combination of prebiotics and probiotics, and therefore have properties of both [9]. As such, they are expected to have a superior effect over separate use of prebiotics or probiotics. Several clinical trials have reported benefits of synbiotics in patients who are obese and those with insulin resistance syndrome, type 2 diabetes, non-alcoholic fatty liver disease, atopic dermatitis, or a variety of gastrointestinal disorders including irritable bowel syndrome and inflammatory bowel disease (reviewed in Markowiak and Śliżweska [9]).

While there is no universal definition for functional foods, the term was first introduced in Japan [10] and has subsequently been defined by several organizations, including the International Food Informational Council, which defines it as 'foods that may provide health benefits beyond basic nutrition' [11] and the American Dietetic Association which defines it as 'foods that provide additional health benefits that may reduce disease risk and/or promote optimal health' [12]. Traditional functional foods include yogurt, natto, kimchi, and many others. Functional foods often contain prebiotics or probiotics, and many contain both. The health benefits of many of these functional foods have been studied and include increased production of SCFAs, improvement of intestinal health, and supporting the growth of bacteria that are considered to provide health benefits, including Lactobacilli and those considered as Lactobacillus, Latilactobacillus, Lentilactobacillus, Lacticaseibacillus, and Lactiplantibacillus [13-16] according to a recently proposed reclassification of the genus Lactobacillus [17].

GoodBiome ${ }^{\mathrm{TM}}$ Foods is a collection of prebiotic and probiotic infused foods that are expected to confer health benefits when consumed since they contain both prebiotics (inulin, xylooligosaccharides, and others) and the probiotic Bacillus subtilis HU58. Bacillus spp. are highly resistant to desiccation and heat and are stable over a wide range of $\mathrm{pHs}$, which make them quite suitable for use as probiotics [18]. B. subtilis HU58 has been shown to be resistant to gastric fluids, to undergo rapid sporulation, and to form biofilms [19]. In clinical trials, treatment with probiotic Bacillus spp. has improved acute diarrhea [20], reduced the incidence of antibiotic-associated diarrhea [21], reduced bloating, pain, and discomfort among patients with irritable bowel syndrome [22-24], and shown some immune effects in healthy volunteers $[25,26]$. Treatment with B. subtilus HU58, in combination with other probiotic strains, has resulted in health benefits in both humans and animals [27-30]. In-vitro studies using probiotic treatments that include B. subtilis HU58 have reported increased levels of SCFAs, increased relative abundance of beneficial microbial families, increased microbial diversity [31], and potential benefits to microbial community recovery after antibiotic exposure [32] with treatment versus untreated control.

This study was conducted to evaluate the effects of repeated intake of three GoodBiome ${ }^{\mathrm{TM}}$ Foods products, as compared to a comparator alternative product on the microbial community activity and the microbial community composition using the mucosal simulator of the human intestinal microbial ecosystem (M-SHIME ${ }^{\circledR}$ ) platform, which is an in-vitro model of the human intestinal tract [33-35] under healthy gut conditions.

\section{Materials and Methods}

\subsection{Test Products and Predigestion}

Three GoodBiome ${ }^{\mathrm{TM}}$ Foods products (Microbiome Labs; Glenview, IL, USA) and one Kodiak Cakes product (Kodiak; Park City, UT, USA) were used as test products. The GoodBiome ${ }^{\mathrm{TM}}$ Foods products included their Lemon Chia Muffin (LCM), Berry Blast Muffin (BBM), and the Oat Spice Mookie (OSM). These products each contain prebiotics, including inulin powder, and the probiotic strain B. subtillis HU58. The Chocolate Chip 
Muffin Cup from Kodiak Cake (KC) was used as the comparator product and did not contain prebiotics or probiotics. The ingredients for each of these products are listed in Supplementary File Text S1.

Each day, the test products were prepared with water using a microwave oven according to the package instructions. Next, $25 \mathrm{~g}$ was predigested using a static digestion method to simulate upper gastrointestinal tract digestion. Predigested products were supplemented into the M-SHIME ${ }^{\circledR}$ system once daily. Predigestion was performed to ensure that all fractions of the test products would reach the colon. The predigestion protocol was based on the consensus protocol developed within a large European framework (COST Action InfoGest) [36] in which the test products were subjected to oral, gastric, and small intestinal incubation. This static digestion protocol was further improved as described by Van den Abbeele et al. [37] by incorporating more accurate $\mathrm{pH}$ profiles together with a simulation of small intestinal absorption by using a dialysis approach to remove small molecules $(<3.5 \mathrm{kDA})$ from the intestinal digests. Oral incubation was conducted in a simulated salivary medium as described by Mackie, et al. [36]. Next, the test products were incubated in gastric medium and a $\mathrm{pH}$ profile from 5.5 to 2.0 was used to mimic in-vivo-fed gastric conditions more closely. Small intestine incubation was then initiated and after $30 \mathrm{~min}$, dialysis was started using a dialysis tube (ZelluTrans/Roth dialysis membrane, regenerated cellulose, molecular weight cut off $3.5 \mathrm{kDa}$ ) submerged in dialysis fluid ( $3.75 \mathrm{~g} / \mathrm{L} \mathrm{NaHCO}_{3} ; \mathrm{pH} 7.0$ ). During the remaining $4.5 \mathrm{~h}$ incubation, the dialysis fluid was replaced every $45 \mathrm{~min}$. The digested and dialyzed material was used to initiate colonic incubation in the Quad-M-SHIME ${ }^{\circledR}$ model.

\subsection{Quad-M-SHIME ${ }^{\circledR}$ Setup}

A Quad-M-SHIME ${ }^{\circledR}$ configuration was used in this study. This configuration utilizes fewer compartments per condition than the standard SHIME ${ }^{\circledR}$ model [33] but allows for the comparison of four different conditions (i.e., test products) in parallel. Each parallel arm consisted of a compartment to simulate the stomach and small intestine, one to simulate the PC, and one to simulate the DC. The stomach/small intestine compartment operated according to the fill-and-draw principle and peristaltic pumps added $140 \mathrm{~mL}$ of SHIME feed at $\mathrm{pH} 3.0$ (to simulate the stomach), which was then incubated for $1.5 \mathrm{~h}$ and followed by the addition of pancreatic and bile liquid $(60 \mathrm{~mL}$ ) at $\mathrm{pH} 6$ (to simulate the small intestine) and an additional $1.5 \mathrm{~h}$ incubation. Next, the intestinal suspension was pumped into the PC compartment ( $\mathrm{pH} 5.6-5.9$; retention time, $20 \mathrm{~h}$; volume, $500 \mathrm{~mL}$ ) then to the DC compartment ( $\mathrm{pH}$ 6.6-6.9; retention time, $32 \mathrm{~h}$; volume, $800 \mathrm{~mL}$ ). The mucosal compartment was simulated in both the PC and DC by the inclusion of mucin-coated beads, as described by Van den Abbeele et al. [38,39]. Two parallel Quad-M-SHIME ${ }^{\circledR}$ configurations were set up; each configuration was inoculated with microbiota isolated from a fecal sample originating from a single healthy adult volunteer who had not been treated with antibiotics within the 6 months prior to sample collection. Rather than pooling the fecal microbiota from the two donors, this approach was employed to allow us to account for interindividual variability when comparing the effects of the four test products.

The Quad-M-SHIME ${ }^{\circledR}$ experiment had three stages: a two-week stabilization period following the inoculation of the colon compartments with the fecal sample, a two-week control period, and a three-week treatment period. The stabilization period allowed the microbial community to colonize different compartments based on the local environmental conditions. During the control period, samples were collected from the PC and DC compartments to determine the baseline microbial community activity and composition of each region. During the treatment period, samples were collected to evaluate the effects of the test products on the microbial activity and community. The Quad-M-SHIME ${ }^{\circledR}$ was fed throughout the study using standard SHIME ${ }^{\circledR}$ feed; during the treatment period, the standard feed was supplemented with the predigested test products. 


\subsection{Microbial Community Activity}

Samples were collected three times per week from each colonic compartment during the control and treatment periods to assess SCFA (acetic acid, propionic acid, and butyric acid), lactate, ammonium, and branched SCFA (isobutyric acid, isovaleric acid, and isocaproic acid) levels. SCFA and branched SCFA levels were assessed using a Nexis GC-2030 Gas Chromatograph (Shimadzu Scientific Instruments, Kyoto, Japan), as described previously [40]. A kit (R-Biopharm, Darmstadt, Germany) was used to determine lactate levels and the levels of ammonium were determined as previously reported in Duysburgh et al. [41].

\subsection{Microbial Community Composition}

Samples from each colon compartment were collected three times per week during the control and treatment periods. Lumen samples were assessed using quantitative shallow shotgun sequencing and mucus samples were assessed using relative shallow shotgun sequencing. The shallow shotgun sequencing method sequences all genomic DNA in the sample and follows a workflow similar to whole genome sequencing; this method was chosen due to the greater genomic coverage and data output. For the shallow shotgun sequencing, DNA samples were quantified using a Qubit 4 fluorometer and Qubit ${ }^{\mathrm{TM}} \mathrm{dsDNA}$ HS Assay Kit (Thermo Fisher Scientific, Waltham, MA, USA). The Nextera XT DNA Library Preparation Kit (Illumina, San Diego, CA, USA) and IDT Unique Dual Indexes were used to prepare DNA libraries (1 ng DNA input); genomic DNA was fragmented with Nextera ST fragmentation enzyme (Illumina). After unique dual indexes were added, samples underwent 12 cycles of PCR to construct libraries, which were purified using AMpure magnetic beads (Beckman Coulter, Brea, CA, USA) followed by elution in QIAGEN EB buffer, and quantification as described above. Unassembled sequencing reads were analyzed directly using the CosmosID-HUB Microbiome Platform (CosmosID Inc., Germantown, MD, USA), as described elsewhere [42-45], for multikingdom microbiome analysis and quantification of organisms' relative abundance. Briefly, the system utilizes curated genome databases and a high-performance datamining algorithm that rapidly disambiguates hundreds of millions of metagenomic sequence reads into the discrete microorganisms engendering the particular sequences. Three million reads were generated from the genomes of the entire microbial community, resulting in semi-quantitative enumeration of the main members of the community (for all microorganisms representing at least $0.01 \%$ of the community). This approach allows examination of the complete composition of the gut microbiota so that modification of the relative abundance of different microbial groups upon exposure to a specific product can be evaluated. Accurate quantification of total bacterial cells in the sample was then accomplished using flow cytometry. Briefly, 10-fold serial dilutions of all samples were prepared in Dulbecco's phosphate-buffered saline (Sigma-Aldrich, Bornem, Belgium); cells were then stained with 0.01-mM SYTO24 (Life Technologies Europe, Merelbeke, Belgium) for $150 \mathrm{~min}$ at $37^{\circ} \mathrm{C}$ in the dark. Samples were analyzed on a BD Facsverse (BD Biosciences, Erembodegem, Belgium) using the high flow rate setting and bacteria were separated from medium debris and signal noise by applying a threshold level of 200 on the SYTO channel. Flowcytometry data were analyzed using FlowJo (Ashland, OR, USA), version 10.5.2. The methods used have been described previously in Vandeputte et al. [46].

\subsection{Statistical Analysis}

A two-tailed homoscedastic t-test was used for comparing the treatment and control periods (community activity and community composition). Comparisons of the different test products were done using a two-tailed paired t-test, as data from samples taken at the same timepoints and from equivalent compartments were compared. To achieve a normal distribution, log-transformed data were used to perform statistical tests of the microbial community composition. A $p$ value of $<0.05$ was considered statistically significant. 


\section{Results}

\subsection{Microbial Community Activity}

Carbohydrate metabolism in the colon results in the production of SCFAs, the most abundant of which are acetate, propionate, and butyrate. Changes in SCFA levels and lactate for Donor A are shown in Figure 1A-D and for Donor B in Figure 1E,F. Acetate levels significantly increased during the treatment period with all test products and in both the PC and DC compartments with the exception of the KC product in the PC for Donor B. For Donor A, the greatest increase in acetate in the PC occurred with supplementation of the OSM (14.1 mM increase, $+94.2 \%$ compared with the control period) and the greatest increase in the DC was with supplementation with the LCM (17.4 mM increase, +56.9\%) (Figure 1A). In Donor B, the greatest increase was observed with OSM supplementation in both the PC and DC (10.8 mM increase, $+43.8 \%$ and $15.1 \mathrm{mM}$ increase, $+42.3 \%$, respectively) (Figure 1E).
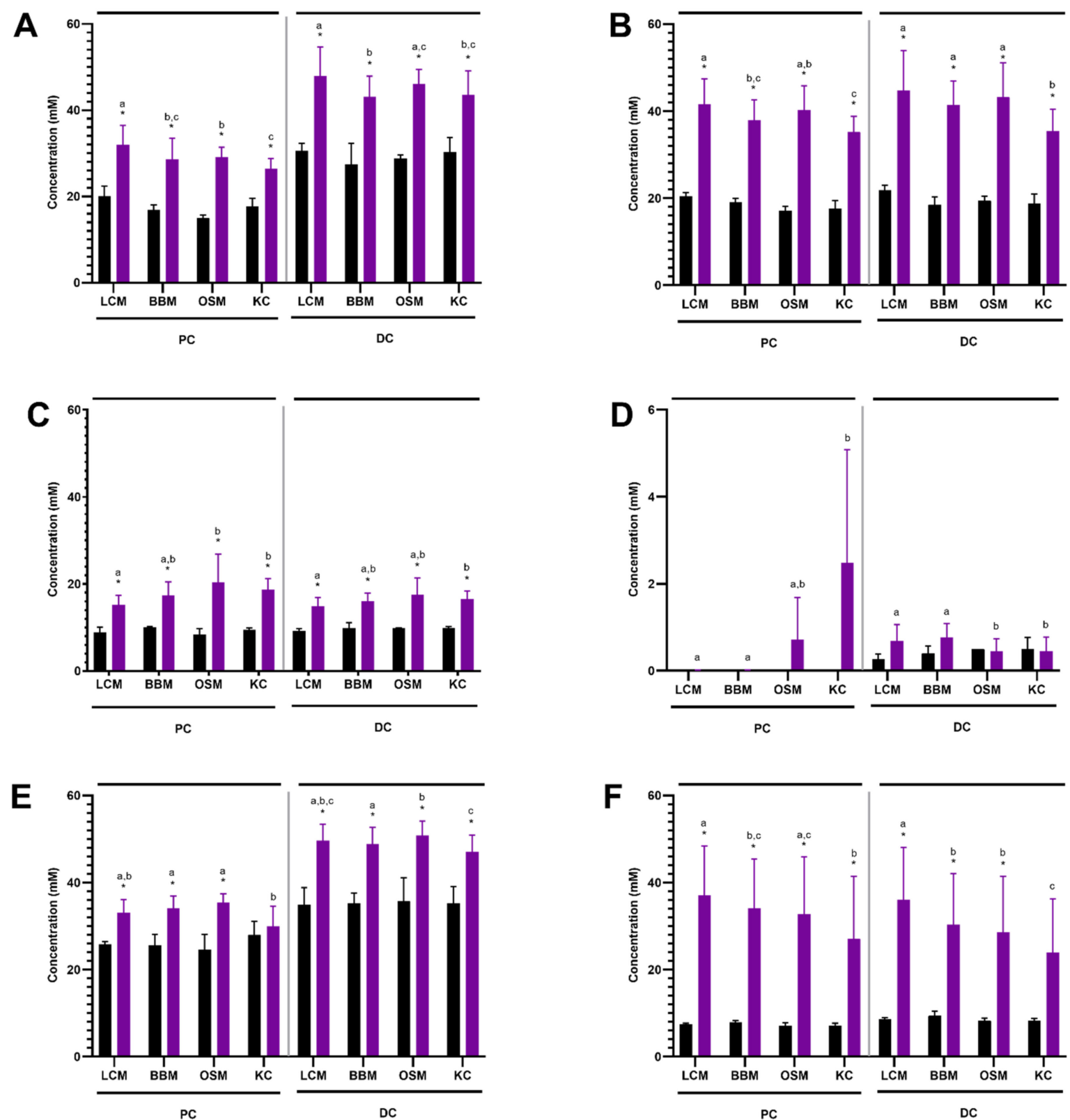

Figure 1. Cont. 

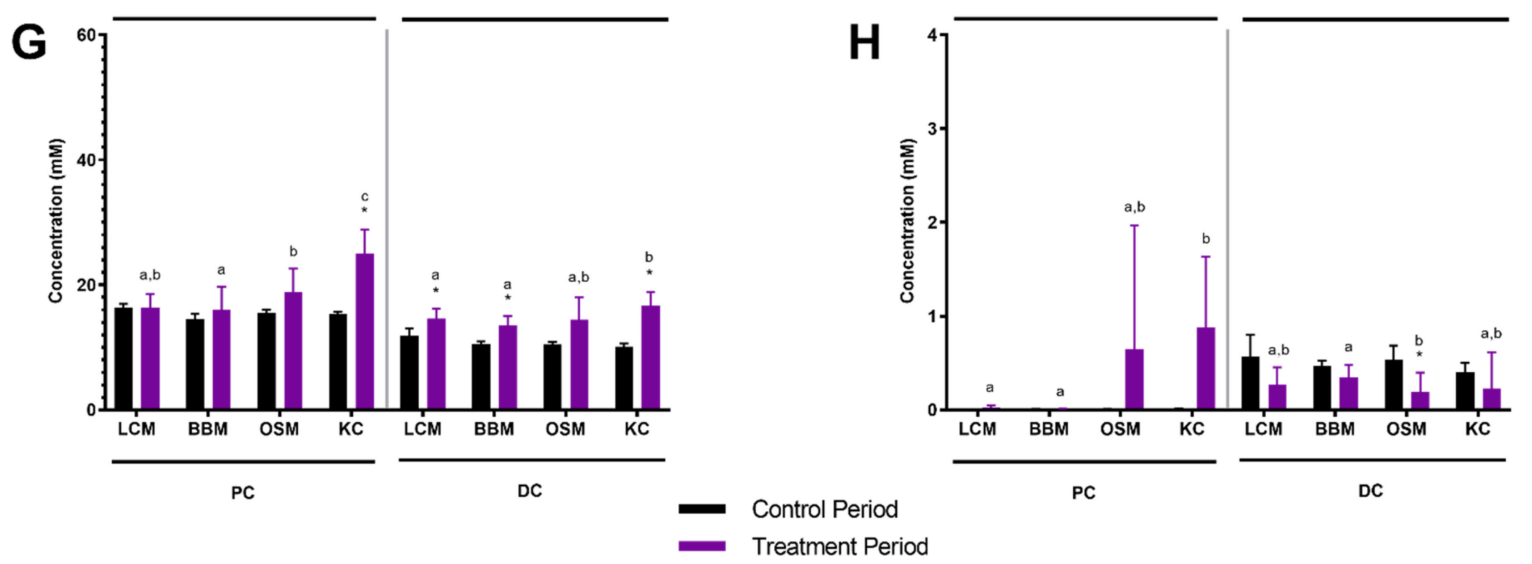

Figure 1. Changes in SCFAs and lactate levels with treatment for (A) Donor A, acetate; (B) Donor A, propionate; (C) Donor A, butyrate; (D) Donor A, lactate; (E) Donor B, acetate; (F) Donor B, propionate; (G) Donor B, butyrate; (H) Donor B, lactate. * Indicates statistically significant differences relative to the control period, while different letters indicate a statistical difference between different treatments, i.e., different letters for different columns indicates that the values in the columns are statistically significantly different while those marked with the same letter are not; $p<0.05$. BBM = Berry Blast Muffin; $\mathrm{DC}=$ distal colon; $\mathrm{KC}=$ Kodiak Cake; $\mathrm{LCM}=$ Lemon Chia Muffin; OSM = Oat Spice Mookie; PC = proximal colon; SCFA $=$ short-chain fatty acid.

Propionate levels during the control period were higher for Donor A than Donor B. As with acetate, all test products significantly increased propionate levels over control levels in both the PC and DC, and for both donors, though the effects were stronger in Donor $\mathrm{B}$, likely due to the lower baseline levels in this donor. Of note, the GoodBiome ${ }^{\mathrm{TM}}$ Food products induced significantly stronger propionic effects when compared to $\mathrm{KC}$ in both colon regions and in both donors, with the exception of BBM in the PC (Figure 1B,F). OSM supplementation had the strongest propionic effects in Donor A (PC, $23.1 \mathrm{mM}$ increase, $+135.1 \%$; DC, $23.8 \mathrm{mM}$ increase, $+122.6 \%$ ). In Donor B, LCM supplementation had the strongest effects (PC, $29.7 \mathrm{mM}$ increase, $+401.4 \%$; DC, $27.4 \mathrm{mM}$ increase $+317.3 \%$ ).

Baseline levels of butyrate were higher in Donor B than Donor A. For Donor A, all test products significantly increased butyrate levels in both the $\mathrm{PC}$ and $\mathrm{DC}$; the strongest effect was observed with OSM supplementation (PC, $12.0 \mathrm{mM}$ increase, $+142.8 \%$; DC, $7.7 \mathrm{mM}$ increase, $+78.3 \%$ ) (Figure $1 \mathrm{C}$ ). For Donor $\mathrm{B}, \mathrm{KC}$ was the only product that significantly enhanced butyrate levels versus the control period in the PC, though all test products, with the exception of the OSM, did so in the DC, where the biggest increase was observed with KC supplementation ( $6.6 \mathrm{mM}$ increase, $+65.3 \%$ ) (Figure 1G). Overall, the significant increase in SCFAs in the DC demonstrates that the test products are fermented not only in the PC, but also in the DC.

Lactate concentrations can fluctuate both as a result of increased production and decreased conversion, as lactate can be converted to butyrate and/or propionate. For both donors, lactate levels were very low throughout the control period and supplementation with test products had little effect (Figure 1D,H).

Ammonium and bSCFAs are produced as a result of protein degradation; changes in the levels of ammonium and bSCFAs from the control to the treatment periods are shown in Figure 2A-D. In our study, all test products resulted in a significant increase in ammonium levels in both the PC and DC and for both donors (Figure 2A,C). Further, bSCFA levels were also significantly increased (Figure 2B,D). Changes in ammonium and bSCFA levels were generally similar among the test products, though, overall, supplementation with $\mathrm{KC}$ resulted in somewhat lower increases in ammonium and bSCFAs than the other test products. 


\section{A}

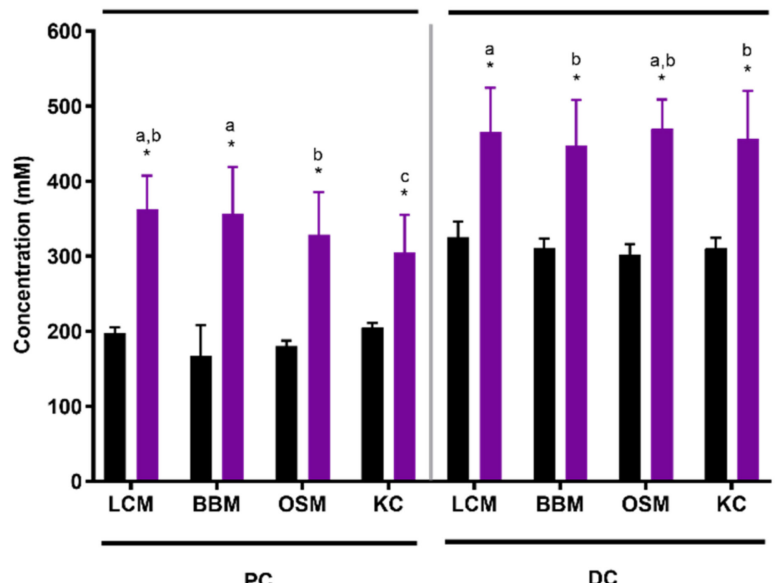

PC

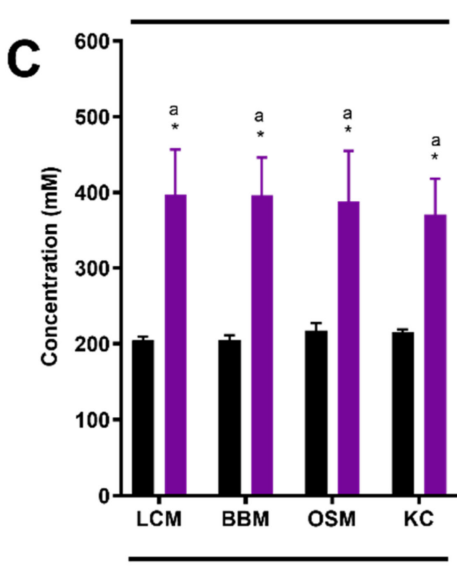

PC
DC

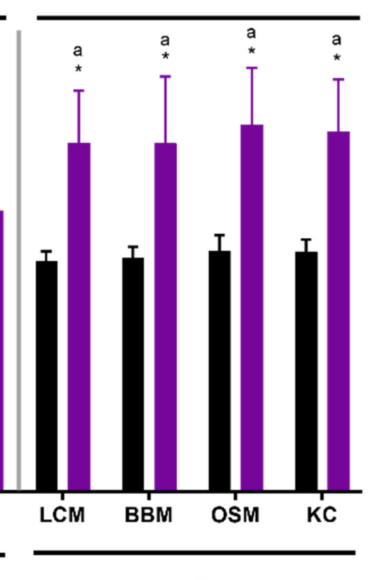

DC
B

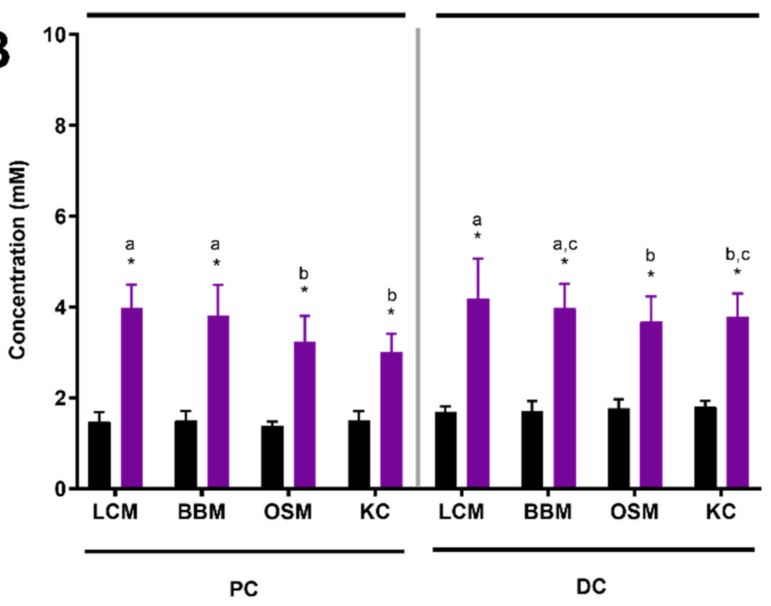

D

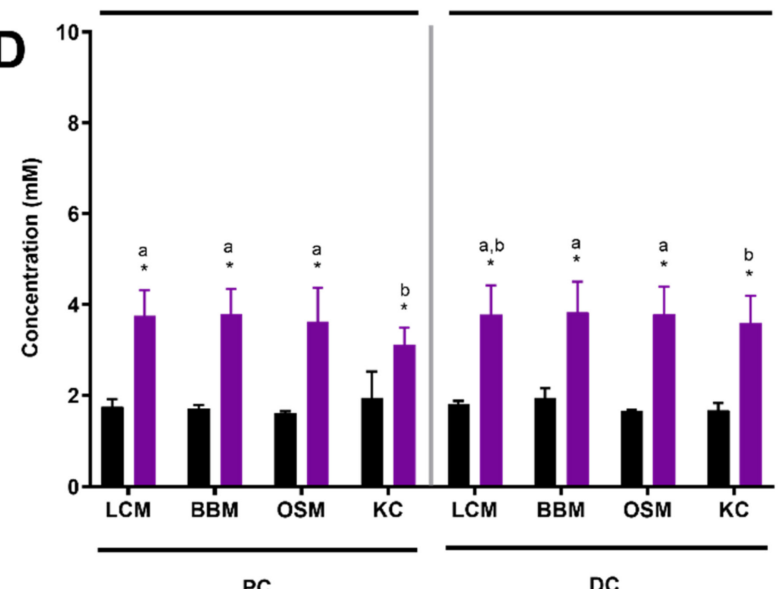

PC

Figure 2. Changes in ammonium and bSCFA levels with treatment for (A) Donor A, ammonium; (B) Donor A, bSCFA; (C) Donor B, ammonium; (D) Donor B, bSCFA. * Indicates statistically significant differences relative to the control period while different letters indicate a statistical difference between different treatments, i.e., different letters for different columns indicates that the values in the columns are statistically significantly different while those marked with the same letter are not; $p<0.05$. BBM = Berry Blast Muffin; DC = distal colon; KC = Kodiak Cake; LCM = Lemon Chia Muffin; OSM = Oat Spice Mookie; $\mathrm{PC}=$ proximal colon .

\subsection{Microbial Community Composition}

The Simpson diversity index revealed that supplementation with the different test products strongly enhanced microbial diversity. The strongest effects were observed in the luminal PC, reaching significance for Donor A, Donor B, and when the values for the two donors were averaged $(p<0.05$ for all) (Table 1$)$. When the values for the two donors were averaged, the LCM was the only test product that resulted in a significant increase in the Simpson diversity index in the lumen and mucus DC compared with the control period while treatment with the other three test products, BBM, OSM, and KC, resulted in a significant increase in the mucosal PC compared with the control period. 
Table 1. Reciprocal Simpson diversity index in the lumen of the proximal colon at the end of the control and treatment periods.

\begin{tabular}{|c|c|c|c|c|c|c|c|c|c|c|}
\hline & & & \multicolumn{2}{|c|}{ LCM } & \multicolumn{2}{|c|}{ BBM } & \multicolumn{2}{|c|}{ OSM } & \multicolumn{2}{|c|}{ KC } \\
\hline & & & $\mathrm{C}$ & TR & $\mathrm{C}$ & TR & $\mathrm{C}$ & TR & $\mathrm{C}$ & TR \\
\hline \multirow{4}{*}{ Donor A } & \multirow{2}{*}{ lumen } & PC & 8.3 & 22.4 * & 8.4 & 19.5 * & 10.3 & $22.4^{*}$ & 9.4 & 21.2 * \\
\hline & & $\mathrm{DC}$ & 18.8 & 23.1 * & 21.9 & 20.3 & 21.5 & 24.8 & 21.2 & 23.3 \\
\hline & \multirow{2}{*}{ mucus } & PC & 18.1 & 25.2 & 13.5 & $25.9 *$ & 16.9 & 20.6 & 15.0 & 21.9 * \\
\hline & & DC & 15.6 & 31.5 & 25.0 & 26.0 & 25.4 & 25.1 & 26.2 & 28.9 \\
\hline \multirow{4}{*}{ Donor B } & \multirow{2}{*}{ lumen } & PC & 5.5 & 22.6 * & 7.1 & $21.5 *$ & 6.8 & $16.8^{*}$ & 3.5 & 20.4 * \\
\hline & & $\mathrm{DC}$ & 15.6 & 21.7 * & 14.3 & 19.4 & 18.1 & 19.2 & 17.8 & 18.7 \\
\hline & \multirow{2}{*}{ mucus } & $\mathrm{PC}$ & 15.8 & 20.3 & 14.7 & 17.6 & 11.8 & $19.1^{*}$ & 10.9 & 17.5 * \\
\hline & & DC & 10.2 & 24.0 * & 11.2 & $22.5 *$ & 11.7 & $26.5^{*}$ & 13.7 & 24.3 * \\
\hline \multirow{4}{*}{$\begin{array}{l}\text { Average of Donors } \\
\qquad \mathrm{A} \text { and } \mathrm{B}\end{array}$} & \multirow{2}{*}{ lumen } & PC & 6.9 & 22.5 * & 7.7 & $20.5 *$ & 8.6 & $19.6^{*}$ & 6.4 & 20.8 * \\
\hline & & DC & 17.2 & 22.4 * & 18.1 & 19.9 & 19.8 & 22.0 & 19.5 & 21.0 \\
\hline & \multirow{2}{*}{ mucus } & PC & 16.9 & 22.7 & 14.1 & $21.7^{*}$ & 14.4 & 19.9 * & 12.9 & 19.7 * \\
\hline & & $\mathrm{DC}$ & 12.9 & 27.8 * & 18.1 & 24.3 & 18.5 & 25.8 & 19.9 & 26.6 \\
\hline
\end{tabular}

The relative abundance of the microbial community at the phylum level for the lumen is shown in Figure 3. In the luminal PC, supplementation resulted in a significant increase $(p<0.05)$ in the relative abundance of Proteobacteria for both donors between the control and treatment periods for all four test products (Figure 3A,B). The relative abundance of Actinobacteria was significantly decreased $(p<0.05)$ with all test products except for the KC for Donor A and with all test products for Donor B. Additionally, the relative abundance of Bacteroidetes was significantly increased $(p<0.05)$ with the KC product in the luminal PC for Donor B. In the luminal DC of Donor A, the relative abundance of Bacteroidetes was significantly decreased $(p<0.05)$ between the control and treatment periods with all test products and the relative abundance of Proteobacteria was significantly increased $(p<0.05)$ for all test products except the BBM (Figure 3C,D). The relative abundances of Verrucomicrobia and Actinobacteria were also significantly increased $(p<0.05)$ with the OSM product. For Donor B, the relative abundance of Actinobacteria was significantly decreased $(p<0.05)$ between the control and treatment periods with all test products, that of Proteobacteria was significantly increased $(p<0.05)$ with LCM, and that of Firmicutes was significantly increased $(p<0.05)$, while Verrucomicrobia and Bacteroidetes were significantly decreased $(p<0.05)$ with the KC product. Overall, there were fewer changes in the relative abundance at the phylum level in the mucosal PC and DC compared with the lumen (Figure S1).

Relative abundance at the family level for the lumen is shown in Figure 4. While the phylum Bacteroidetes did not significantly expand in the luminal PC, at the species level Bacteroidaceae tended to decrease while Tannerellaceae and Rikenellaceae tended to increase from the control period to the treatment period for each of the test products and with both donors (Figure 4A,B). Also in the luminal PC, the expansion of Proteobacteria after supplementation with the test products was largely due to the expansion of Enterobacteriaceae. The expansion of Proteobacteria in the luminal DC with supplementation was also largely due to Enterobacteriaceae with all test products; other changes between the control and treatment period were variable among the test products (Figure 4C,D). There were few changes at the species level in the mucosal PC and DC (Figure S2). 


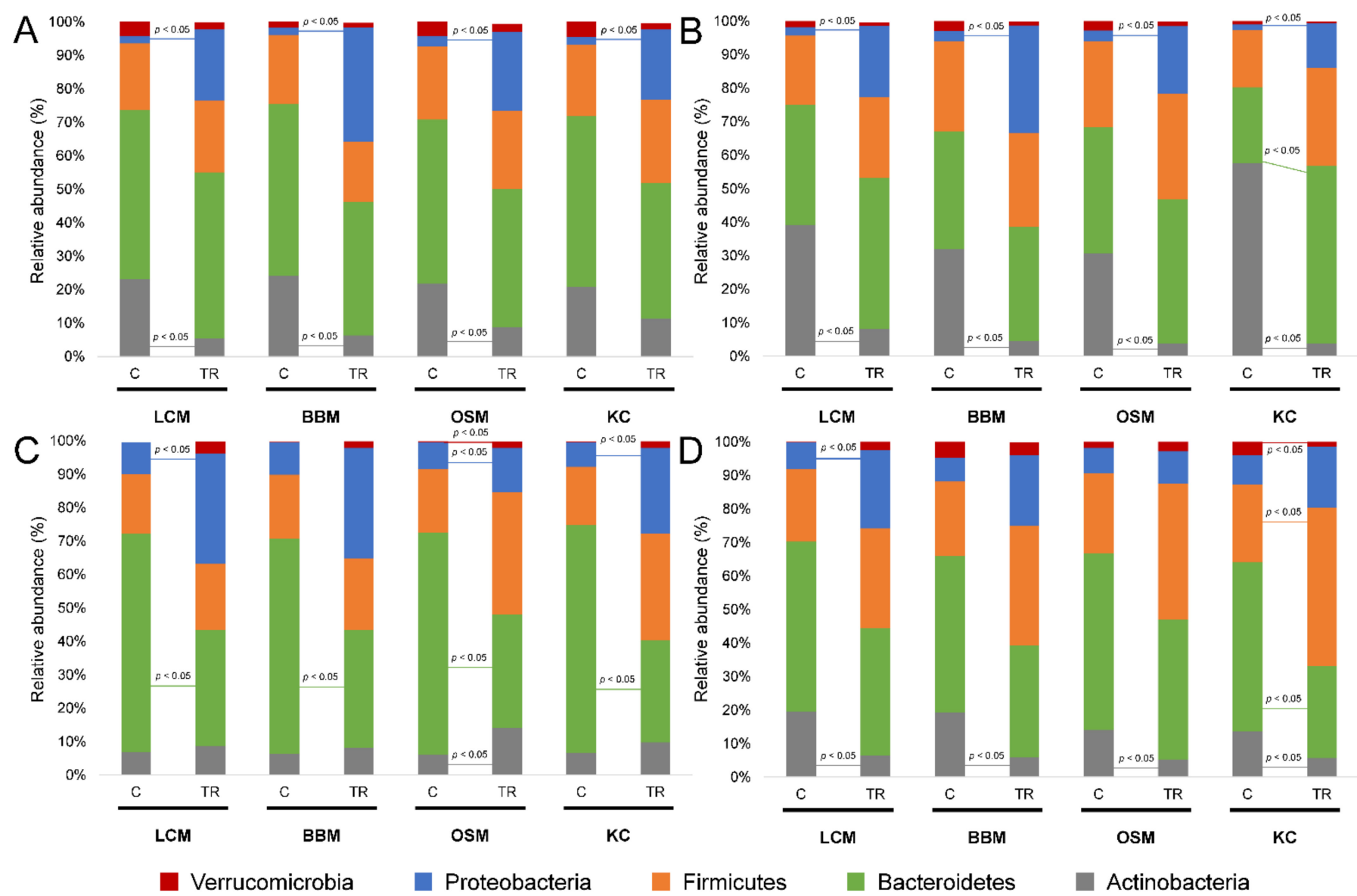

Figure 3. Changes in microbial community composition (relative abundance) at the phylum level in the luminal PC and DC for (A) Donor A, luminal PC; (B) Donor B, luminal PC; (C) Donor A, luminal DC; (D) Donor B, luminal DC. BBM = berry blast muffin; $\mathrm{C}=$ control period; $\mathrm{DC}=$ distal colon; $\mathrm{KC}=$ Kodiak cake; $\mathrm{LCM}=$ lemon chia muffin; OSM = oat spice mookie; $\mathrm{PC}=$ proximal colon; $\mathrm{TR}=$ treatment period.
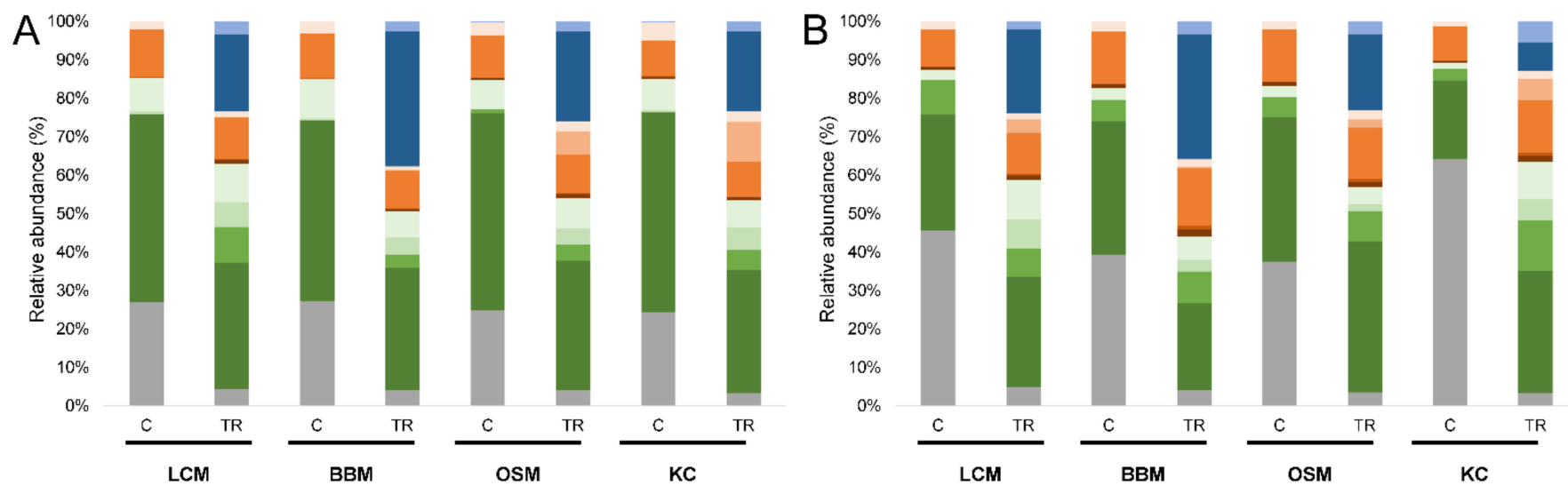

Figure 4. Cont. 


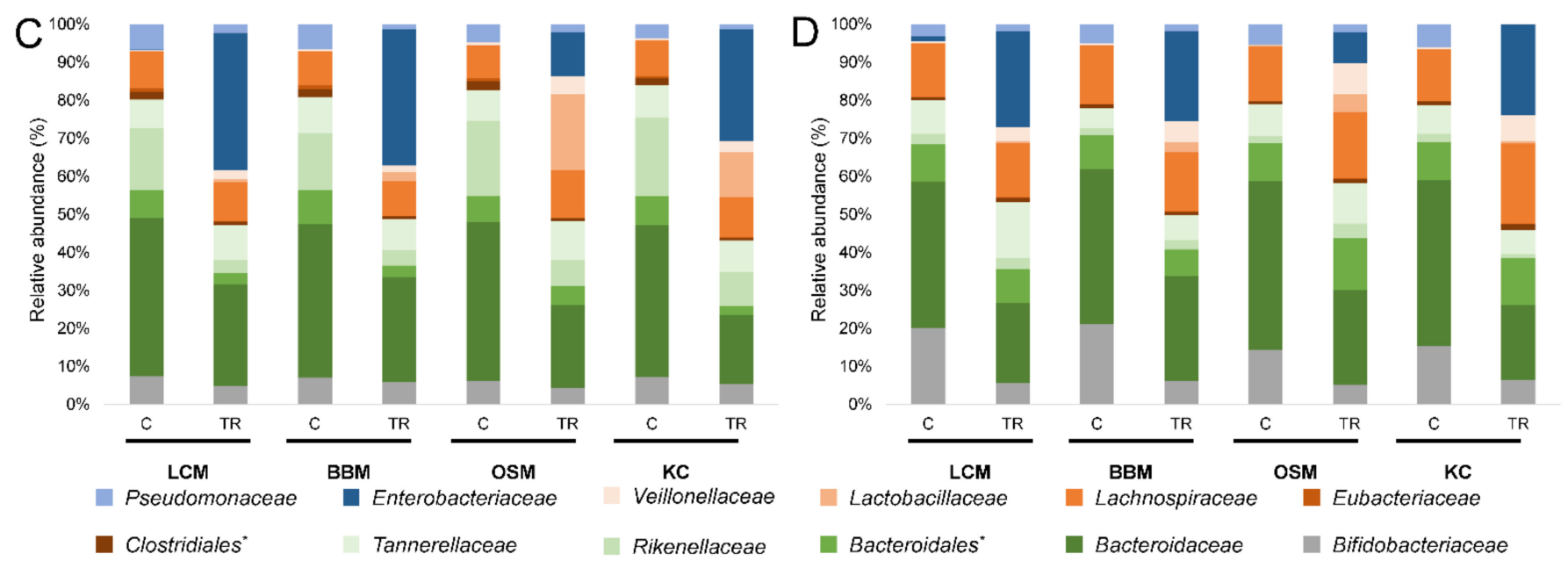

Figure 4. Changes in microbial community composition (relative abundance) at the family level in the luminal PC and DC for (A) Donor A, luminal PC; (B) Donor B, luminal PC; (C) Donor A, luminal DC; (D) Donor B, luminal DC. * Unidentified family. BBM = Berry Blast Muffin; $\mathrm{C}=$ control period; $\mathrm{DC}=$ distal colon; $\mathrm{KC}=$ Kodiak Cake; $\mathrm{LCM}=$ Lemon Chia Muffin; $\mathrm{OSM}=$ Oat Spice Mookie; PC = proximal colon; TR = treatment period.

\section{Discussion}

Overall, all four of the products tested resulted in an increase in SCFAs for the treatment period versus the control period. This was generally true for both donors, though there were some exceptions, likely the result of donor specific differences in baseline microbial community composition. The test products had little effect on lactate levels. Ammonium and bSCFA levels were also significantly increased in the treatment versus control period in the PC and DC compartments for all test products and both donors. All test products resulted in a significant increase in the Simpson diversity index in the treatment versus control periods in the luminal PC, supplementation with the LCM resulted in a significant increase for both the luminal and mucosal DC, and supplementation with the other three test products resulted in a significant increase in the mucosal PC.

The significant increase in SCFAs in both the PC and DC is noteworthy, as it indicates that the test products are being fermented in both compartments. The propiogenic response was significantly stronger with the GoodBiome ${ }^{\mathrm{TM}}$ Foods products than with the comparator, indicating that the addition of prebiotics and B. subtillis HU58 may be responsible for the increased propiogenic response. This response was strongest for donor A with the OSM product and for donor B with the LCM product. Although all test products resulted in increased acetate production, the OSM had the strongest overall effect. In donor A, an increase in butyrate was observed for all test products with the largest increase in butyrate production occurring with the OSM; butyrate production was only significantly increased with $\mathrm{KC}$ for donor $\mathrm{B}$.

Increased microbial diversity in the human gut is generally considered beneficial. For example, a recent study in humans reported that long term weight gain was associated with a significantly lower Simpson diversity index compared with individuals who did not experience long term weight gain [47]. Dietary fiber intake was also associated with less weight gain. Another study found that higher $\alpha$ diversity in the gut microbiome, along with more butyrate-producing species, were associated with a lower incidence of type 2 diabetes and lower rates of insulin resistance among non-diabetic individuals [48]. Therefore, we consider the increase in the Simpson diversity index observed with the test products in this in vitro model to be encouraging regarding their potential to impart beneficial effects on the human gut.

The significant increase in Proteobacteria levels in the PC for both donors and with all test products indicates the involvement of this phylum in the primary fermentation of these products. The increase in Proteobacteria was largely due to an increase in the 
Enterobacteriaceae family. While Enterobacteriaceae are present both as resident and transient members of the healthy human microbiome [49,50], they are considered symbionts and have the potential to be harmful as some members of this family are pathogenic. Therefore, the potential negative health effects of an increase in Enterobacteriaceae must be taken into consideration and addressed in future studies. While the Bacteroidetes phylum did not increase significantly, there were changes in the microbial composition at the family level. At the expense of Bacteroidaceae, enrichment was observed for Rikenellaceae and Tannerellaceae, which are a group of primary substrate degraders that produce mainly succinate and acetate [51]. This may partially explain the increases in propionate, as succinate can be converted to propionate [52], and the increases in acetate that were observed, particularly in the PC. Increases in Lactobacillaceae were observed with all test products, but particularly with the OSM and KC. Interestingly, we did not observe an increase in the production of lactate after exposure to the test products, despite the enrichment of this lactate-producing family. This may have been due to an increase lactate consumption, as it is fermented to propionate and acetate [53], which were both increased upon supplementation with the test products.

Overall, the OSM seemed to have the greatest presumed beneficial effects on the microbial community activity, though all GoodBiome ${ }^{\mathrm{TM}}$ Foods products induced changes in this in vitro M-SHIME ${ }^{\circledR}$ model that are thought to be beneficial to the human gut. The comparator product, $\mathrm{KC}$, also demonstrated some of those changes, though the overall effect was weaker compared with the GoodBiome ${ }^{\mathrm{TM}}$ Foods products.

Our study had several limitations. First, as this was an in-vitro study, the results cannot directly translate to a biological response. As such, in-vivo studies are needed to evaluate whether the test products have biological activity in humans. Additionally, our study was conducted using the microbiota of only two donors. While most results were consistent between the two donors, several outcomes, such as the effect of the test products on butyrate production, were, in fact, donor dependent, highlighting the need for further testing in a greater variety of donors. Finally, the donors in our study were healthy and therefore it is unclear what effects the test products may have on the microbiome of donors with gastrointestinal disease, such as inflammatory bowel disease or colitis.

\section{Conclusions}

Both the GoodBiome ${ }^{\mathrm{TM}}$ Foods test products, containing prebiotics and B. subtillis HU58, and the comparator product resulted in an increase in SCFA production and an increase in microbial community diversity during the treatment period versus the control period in an in vitro model of the healthy human intestinal tract. The promising results of this study warrant further study of GoodBiome ${ }^{\mathrm{TM}}$ Foods products as potential functional foods in humans to determine whether regular consumption may result in beneficial effects, such as increased SCFA production or increase microbial community diversity in the gut.

Supplementary Materials: The following are available online at https:/ / www.mdpi.com/article/10 .3390/app112411963/s1, Supplementary File Text S1: Test product ingredients, Figure S1: Changes in microbial community composition at the phylum level in the mucosal PC and DC, Figure S2: Changes in microbial community composition at the family level in the mucosal PC and DC.

Author Contributions: Conceptualization, M.M., T.B., K.K. and A.Y.; methodology, M.M., T.B., K.K. and A.Y.; formal analysis, M.M. and S.B.; data curation, M.M.; writing-original draft preparation, S.B.; writing-review and editing, M.M., T.B., K.K. and A.Y.; visualization, S.B. All authors have read and agreed to the published version of the manuscript.

Funding: This study and the article processing charges were funded by Microbiome Labs.

Institutional Review Board Statement: Fecal samples of the donors were collected according to the ethical approval of the University Hospital Ghent (reference number B670201836585).

Informed Consent Statement: Informed consent was obtained from all fecal donors. 
Data Availability Statement: The data presented in this study are available on request from the corresponding author.

Conflicts of Interest: T.B., K.K. and A.Y. are employees of Microbiome Labs. S.B. received payment from Microbiome Labs. The funders were involved in the design of the study, in the writing of the manuscript, and in the decision to publish the results.

\section{References}

1. Gibson, G.R.; Hutkins, R.; Sanders, M.E.; Prescott, S.L.; Reimer, R.A.; Salminen, S.J.; Scott, K.; Stanton, C.; Swanson, K.S.; Cani, P.D.; et al. Expert consensus document: The International Scientific Association for Probiotics and Prebiotics (ISAPP) consensus statement on the definition and scope of prebiotics. Nat. Rev. Gastroenterol. Hepatol. 2017, 14, 491-502. [CrossRef] [PubMed]

2. Roberfroid, M.B. Health benefits of non-digestible oligosaccharides. Adv. Exp. Med. Biol. 1997, 427, 211-219. [CrossRef] [PubMed]

3. Davani-Davari, D.; Negahdaripour, M.; Karimzadeh, I.; Seifan, M.; Mohkam, M.; Masoumi, S.J.; Berenjian, A.; Ghasemi, Y. Prebiotics: Definition, Types, Sources, Mechanisms, and Clinical Applications. Foods 2019, 8, 92. [CrossRef] [PubMed]

4. Marteau, P. Probiotics, prebiotics, synbiotics: Ecological treatment for inflammatory bowel disease? Gut 2006, 55, 1692-1693. [CrossRef]

5. Marteau, P.; Flourie, B.; Cherbut, C.; Correze, J.L.; Pellier, P.; Seylaz, J.; Rambaud, J.C. Digestibility and bulking effect of ispaghula husks in healthy humans. Gut 1994, 35, 1747-1752. [CrossRef]

6. Stewart, M.L.; Timm, D.A.; Slavin, J.L. Fructooligosaccharides exhibit more rapid fermentation than long-chain inulin in an in vitro fermentation system. Nutr. Res. 2008, 28, 329-334. [CrossRef]

7. Pyne, D.B.; West, N.P.; Cox, A.J.; Cripps, A.W. Probiotics supplementation for athletes-Clinical and physiological effects. Eur. J. Sport Sci. 2015, 15, 63-72. [CrossRef]

8. Thomas, C.M.; Versalovic, J. Probiotics-host communication: Modulation of signaling pathways in the intestine. Gut Microbes 2010, 1, 148-163. [CrossRef]

9. Markowiak, P.; Slizewska, K. Effects of Probiotics, Prebiotics, and Synbiotics on Human Health. Nutrients 2017, 9, 1021. [CrossRef]

10. Siro, I.; Kapolna, E.; Kapolna, B.; Lugasi, A. Functional food. Product development, marketing and consumer acceptance-A review. Appetite 2008, 51, 456-467. [CrossRef]

11. 2011 Functional Foods/Foods for Health Consumer Trending Survey. Available online: https://foodinsight.org/2011-functionalfoods-foods-for-health-consumer-trending-survey/ (accessed on 9 August 2021).

12. Hasler, C.M.; Brown, A.C.; American Dietetic, A. Position of the American Dietetic Association: Functional foods. J. Am. Diet. Assoc. 2009, 109, 735-746. [CrossRef]

13. Cao, Z.H.; Green-Johnson, J.M.; Buckley, N.D.; Lin, Q.Y. Bioactivity of soy-based fermented foods: A review. Biotechnol. Adv. 2019, 37, 223-238. [CrossRef]

14. Terada, S.; Yamamoto, M.; Yoshimura, E. Effect of the Fermented Soybean Product "Natto" on the Composition and Metabolic Activity of the Human Fecal Flora. Jpn. J. Food Microbiol. 1999, 16, 221-230. [CrossRef]

15. Dimidi, E.; Cox, S.R.; Rossi, M.; Whelan, K. Fermented Foods: Definitions and Characteristics, Impact on the Gut Microbiota and Effects on Gastrointestinal Health and Disease. Nutrients 2019, 11, 1806. [CrossRef]

16. Kok, C.R.; Hutkins, R. Yogurt and other fermented foods as sources of health-promoting bacteria. Nutr. Rev. 2018, 76, 4-15. [CrossRef]

17. Zheng, J.; Wittouck, S.; Salvetti, E.; Pranz, C.M.A.P.; Harris, H.M.B.; Mattarelli, P.; O’Toole, P.W.; Pot, B.; Vandamme, P.; Walter, J.; et al. A taxonomic note on the genus Lactobacillus: Description of 23 novel genera, emended description of the genus Lactobacillus Beijerinck 1901, and union of Lactobacillaceae and Leuconostocaceae. Int. J. Syst. Evol. Microbiol. 2020, 70, $2782-2858$. [CrossRef]

18. Cutting, S.M. Bacillus probiotics. Food Microbiol. 2011, 28, 214-220. [CrossRef]

19. Permpoonpattana, P.; Hong, H.A.; Khaneja, R.; Cutting, S.M. Evaluation of Bacillus subtilis strains as probiotics and their potential as a food ingredient. Benef. Microbes 2012, 3, 127-135. [CrossRef]

20. Sudha, R.M.; Bhonagiri, S. Efficacy of Bacillus coagulans strain Unique IS-2 in the treatment of patients with acute diarrhea. Int. J. Probiotics Prebiotics 2012, 7, 33-37.

21. Horosheva, T.V.; Vodyanoy, V.; Sorokulova, I. Efficacy of Bacillus probiotics in prevention of antibiotic-associated diarrhoea: A randomized, double-blind, placebo-controlled clinical trial. JMM Case Rep. 2014, 1, e004036. [CrossRef]

22. Majeed, M.; Majeed, S.; Nagabhushanam, K.; Natarajan, S.; Sivakumar, A.; Ali, F. Evaluation of the stability of Bacillus coagulans MTCC 5856 during processing and storage of functional foods. Int. J. Food Sci. Technol. 2016, 51, 894-901. [CrossRef]

23. Urgesi, R.; Casale, C.; Pistelli, R.; Rapaccini, G.L.; De Vitis, I. A randomized double-blind placebo-controlled clinical trial on efficacy and safety of association of simethicone and Bacillus coagulans (Colinox ${ }^{\circledR}$ ) in patients with irritable bowel syndrome. Eur. Rev. Med. Pharmacol. Sci. 2014, 18, 1344-1353.

24. Madempudi, R.S.; Ahire, J.J.; Neelamraju, J.; Tripathi, A.; Nanal, S. Randomized clinical trial: The effect of probiotic Bacillus coagulans Unique IS2 vs. placebo on the symptoms management of irritable bowel syndrome in adults. Sci. Rep. 2019, 9, 12210. [CrossRef] 
25. Lefevre, M.; Racedo, S.M.; Ripert, G.; Housez, B.; Cazaubiel, M.; Maudet, C.P.; Jüsten, P.; Marteau, P.; Urdaci, M.C. Probiotic strain Bacillus subtilis CU1 stimulates immune system of elderly during common infectious disease period: A randomized, double-blind placebo-controlled study. Immun. Ageing 2015, 12, 24. [CrossRef]

26. Nyangale, E.P.; Farmer, S.; Cash, K.; Chernoff, D.; Gibson, G.R. Bacillus coagulans GBI-30, 6086 modulates Faecalibacterium prausnitziiin older men and women. J. Nutr. 2015, 145, 1446-1452. [CrossRef]

27. Catinean, A.; Neag, M.A.; Krishnan, K.; Muntean, D.M.; Bocsan, C.I.; Pop, R.M.; Mitre, A.O.; Melincovici, C.S.; Buzoianu, A.D. Probiotic Bacillus spores together with amino acids and immunoglobulins exert protective effects on a rat model of ulcerative colitis. Nutrients 2020, 12, 3607. [CrossRef]

28. Catinean, A.; Neag, A.M.; Nita, A.; Buzea, M.; Buzoianu, A.D. Bacillus spp. spores-a promising treatment option for patients with irritable bowel syndrome. Nutrients 2019, 11, 1968. [CrossRef] [PubMed]

29. McFarlin, B.K.; Henning, A.L.; Bowman, E.M.; Gary, M.A.; Carbajal, K.M. Oral spore-based probiotic supplementation was associated with reduced incidence of post-prandial dietary endotoxin, triglycerides, and disease risk biomarkers. World $J$. Gastrointest. Pathophysiol. 2017, 8, 117-126. [CrossRef] [PubMed]

30. Neag, M.A.; Catinean, A.; Muntean, D.M.; Pop, M.R.; Bocsan, C.I.; Botan, E.C.; Buzoianu, A.D. Probiotic Bacillus spores protect against acetaminophen induced acute liver injury in rats. Nutrients 2020, 12, 632. [CrossRef] [PubMed]

31. Marzorati, M.; Van den Abbeele, P.; Bubeck, S.; Bayne, T.; Krishnan, K.; Young, A. Treatment with a spore-based probiotic containing five strains of Bacillus induced changes in the metabolic activity and community composition of the gut microbiota in a SHIME ${ }^{\circledR}$ model of the human gastrointestinal system. Food Res. Int. 2021, 149, 110676. [CrossRef] [PubMed]

32. Marzorati, M.; Van den Abbeele, P.; Bubeck, S.S.; Bayne, T.; Krishnan, K.; Young, A.; Mehta, D.; DeSouza, A. Bacillus subtilis HU58 and Bacillus coagulans SC208 probiotics reduced the effects of antibiotic-induced gut microbiome dysbiosis in an M-SHIME ${ }^{\circledR}$ model. Microorganisms 2020, 8, 1028. [CrossRef]

33. Molly, K.; Vande Woestyne, M.; Verstraete, W. Development of a 5-step multi-chamber reactor as a simulation of the human intestinal microbial ecosystem. Appl. Microbiol. Biotechnol. 1993, 39, 254-258. [CrossRef]

34. Possemiers, S.; Verthe, K.; Uyttendaele, S.; Verstraete, W. PCR-DGGE-based quantification of stability of the microbial community in a simulator of the human intestinal microbial ecosystem. FEMS Microbiol. Ecol. 2004, 49, 495-507. [CrossRef]

35. Van den Abbeele, P.; Grootaert, C.; Marzorati, M.; Possemiers, S.; Verstraete, W.; Gerard, P.; Rabot, S.; Bruneau, A.; El Aidy, S.; Derrien, M.; et al. Microbial community development in a dynamic gut model is reproducible, colon region specific, and selective for Bacteroidetes and Clostridium cluster IX. Appl. Environ. Microbiol. 2010, 76, 5237-5246. [CrossRef]

36. Mackie, A.; Rigby, N. InfoGest Consensus Method. In The Impact of Food Bioactives on Health: In Vitro and Ex Vivo Models; Verhoeckx, K., Cotter, P., Lopez-Exposito, I., Kleiveland, C., Lea, T., Mackie, A., Requena, T., Swiatecka, D., Wichers, H., Eds.; Springer: Cham, Switzerland, 2015; pp. 13-22.

37. Van den Abbeele, P.; Kamil, A.; Fleige, L.; Chung, Y.; De Chavez, P.; Marzorati, M. Different Oat Ingredients Stimulate Specific Microbial Metabolites in the Gut Microbiome of Three Human Individuals in Vitro. ACS Omega 2018, 3, 12446-12456. [CrossRef]

38. Van den Abbeele, P.; Belzer, C.; Goossens, M.; Kleerebezem, M.; De Vos, W.M.; Thas, O.; De Weirdt, R.; Kerckhof, F.M.; Van de Wiele, T. Butyrate-producing Clostridium cluster XIVa species specifically colonize mucins in an in vitro gut model. ISME J. 2013, 7, 949-961. [CrossRef]

39. Van den Abbeele, P.; Roos, S.; Eeckhaut, V.; MacKenzie, D.A.; Derde, M.; Verstraete, W.; Marzorati, M.; Possemiers, S.; Vanhoecke, B.; Van Immerseel, F.; et al. Incorporating a mucosal environment in a dynamic gut model results in a more representative colonization by lactobacilli. Microb. Biotechnol. 2012, 5, 106-115. [CrossRef]

40. Ghyselinck, J.; Verstrepen, L.; Moens, F.; Van den Abbeele, P.; Said, J.; Smith, B.; Bjarnason, I.; Basit, A.W.; Gaisford, S. A 4-strain probiotic supplement influences gut microbiota composition and gut wall function in patients with ulcerative colitis. Int. J. Pharm. $X$ 2020, 587, 119648. [CrossRef]

41. Duysburgh, C.; Van den Abbeele, P.; Krishnan, K.; Bayne, T.F.; Marzorati, M. A synbiotic concept containing spore-forming Bacillus strains and a prebiotic fiber blend consistently enhanced metabolic activity by modulation of the gut microbiome in vitro. Int. J. Pharm. X 2019, 1, 100021. [CrossRef]

42. Ottesen, A.; Ramachandran, P.; Reed, E.; White, J.R.; Hasan, N.; Subramanian, P.; Ryan, G.; Jarvis, K.; Grim, C.; Daquiqan, N.; et al. Enrichment dynamics of Listeria monocytogenes and the associated microbiome from naturally contaminated ice cream linked to a listeriosis outbreak. BMC Microbiol. 2016, 16, 275. [CrossRef]

43. Ponnusamy, D.; Kozlova, E.V.; Sha, J.; Erova, T.E.; Azar, S.R.; Fitts, E.C.; Kirtley, M.L.; Tiner, B.L.; Andersson, J.A.; Grim, C.J.; et al. Cross-talk among flesh-eating Aeromonas hydrophila strains in mixed infection leading to necrotizing fasciitis. Proc. Natl. Acad. Sci. USA 2016, 113, 722-727. [CrossRef]

44. Hasan, N.A.; Young, B.A.; Minard-Smith, A.T.; Saeed, K.; Li, H.; Heizer, E.M.; McMillan, N.J.; Isom, R.; Abdullah, A.S.; Bornman, D.M.; et al. Microbial community profiling of human saliva using shotgun metagenomic sequencing. PLoS ONE 2014, 9, e97699. [CrossRef]

45. Lax, S.; Smith, D.P.; Hampton-Marcell, J.; Owens, S.M.; Handley, K.M.; Scott, N.M.; Gibbons, S.M.; Larsen, P.; Shogan, B.D.; Weiss, S.; et al. Longitudinal analysis of microbial interaction between humans and the indoor environment. Science 2014, 345, 1048-1052. [CrossRef] 
46. Vandeputte, D.; Kathagen, G.; D’Hoe, K.; Vieira-Silva, S.; Valles-Colomer, M.; Sabino, J.; Wang, J.; Tito, R.Y.; De Commer, L.; Darzi, Y.; et al. Quantitative microbiome profiling links gut community variation to microbial load. Nature 2017, 551, 507-511. [CrossRef]

47. Menni, C.; Jackson, M.A.; Pallister, T.; Steves, C.J.; Spector, T.D.; Valdes, A.M. Gut microbiome diversity and high-fibre intake are related to lower long-term weight gain. Int. J. Obes. 2017, 41, 1099-1105. [CrossRef]

48. Chen, Z.; Radjabzadeh, D.; Chen, L.; Kurilshikov, A.; Kavousi, M.; Ahmadizar, F.; Ikram, M.A.; Uitterlinden, A.G.; Zhernakova, A.; Fu, J.; et al. Association of Insulin Resistance and Type 2 Diabetes With Gut Microbial Diversity: A Microbiome-Wide Analysis From Population Studies. JAMA Netw. Open 2021, 4, e2118811. [CrossRef]

49. Martinson, J.N.V.; Pinkham, N.V.; Peters, G.W.; Cho, H.; Heng, J.; Rauch, M.; Broadaway, S.C.; Walk, S.T. Rethinking gut microbiome residency and the Enterobacteriaceae in healthy human adults. ISME J. 2019, 13, 2306-2318. [CrossRef]

50. Martinson, J.N.V.; Walk, S.T. Escherichia coli Residency in the Gut of Healthy Human Adults. EcoSal Plus 2020, 9, 9. [CrossRef]

51. Krieg, N.R.; Staley, J.T.; Brown, D.R.; Hedlund, B.P.; Paster, B.J.; Ward, N.L.; Ludwig, W.; Whitman, W.B. Bergey's Manual of Systemic Bacteriology, 2nd ed.; Springer: New York, NY, USA, 2012; Volume 4.

52. Macy, J.M.; Ljungdahl, L.G.; Gottschalk, G. Pathway of succinate and propionate formation in Bacteroides fragilis. J. Bacteriol. 1978, 134, 84-91. [CrossRef]

53. Seeliger, S.; Janssen, P.H.; Schink, B. Energetics and kinetics of lactate fermentation to acetate and propionate via methylmalonylCoA or acrylyl-CoA. FEMS Microbiol. Lett. 2002, 211, 65-70. [CrossRef] 